\title{
CD207+/langerin positive dendritic cells in invasive and in situ cutaneous malignant melanoma
}

\author{
Grzegorz Dyduch ${ }^{1}$, Katarzyna E. Tyrak², Anna Glajcar ${ }^{1}$, Joanna Szpor ${ }^{1}$, Krzysztof Okoń ${ }^{1}$ \\ ${ }^{1}$ Chair of Pathomorphology, Jagiellonian University Medical College, Krakow, Poland \\ ${ }^{2}$ II Chair of Internal Medicine, Jagiellonian University Medical College, Krakow, Poland
}

Adv Dermatol Allergol 2017; XXXIV (3): 233-239

DOI: https://doi.org/10.5114/ada.2017.67845

\begin{abstract}
Introduction: Dendritic cells are crucial for cutaneous immune response. Their role in melanoma progression is however a matter of controversy.

Material and methods: The number of dendritic cells within epidermis and in peri- and intratumoral location was analyzed using CD207 immunostain in 17 cases of in situ and 25 case of invasive melanoma.

Results: Average peritumoral CD207+ cells count was 22.88 for all cases, 17.94 for in situ lesions and 26.24 for invasive cases. Average epidermal CD207+ cells count was 164.47 for all cases, 183.00 for in situ lesions and 150.78 - for invasive cases. In case of invasive melanomas, peritumoral CD207+ cells count was positively correlated with Breslow stage $(R=0.59)$ mitotic activity within the tumor $(R=0.62)$. Invasive cases with regression showed higher intratumoral and epidermal CD207+ cells count than the ones without (275.00 vs. 95.32 and 173.20 vs. 148.35) but lower peritumoral CD207+ cells count (17.60 vs. 27.26). Invasive cases with ulceration showed higher intratumoral and peritumoral CD207+ cells count than the ones without ulceration (220.08 vs. 55.67 and 44.17 vs. 9.69).

Conclusions: CD207+ cells play a role in both progression and regression of melanoma but their exact role needs further studies.
\end{abstract}

Key words: malignant melanoma, dendritic cells.

\section{Introduction}

The incidence of cutaneous malignant melanoma (CMM) in Europe has steadily increased over the last decades [1-3]. In 2012, CMM ranked the seventh among the most frequently diagnosed malignancies in the European Union, constituting 3\% of all new cancer cases (excluding non-melanoma skin cancers) [4]. Moreover, in many EU countries the mortality of CMM has been continuously increasing [5] and despite developments in melanoma treatment, including BRAF or MEK inhibitors as well as anti-CTLA4 monoclonal antibodies [6, 7], clinicians are still powerless in the most advanced cases. Consequently, there is still much to be discovered and scientists should go further in searching for new prognostic, predictive and therapeutic tools.

In recent years, many authors have demonstrated that cancer development and progression is, to some extent, controlled by the host's immune system [8-11], which suggests that immunological biomarkers might be good indicators of patient's outcome. As CMM is consid- ered to be a highly immunogenic tumour [12], the utilisation of immune cells as prognostic determinants seems to represent a very promising approach. Recently, there has also been a growing interest in therapeutic potential of immune cell-based vaccines against various human malignancies [13-16], including CMM [17, 18]. Especially dendritic cells (DCs) have been widely investigated, since they are believed to be capable of inducing tumour-specific immunity [19] and, thus, could be used as prognostic biomarkers as well as potential therapeutic targets.

Dendritic cells are the most potent, highly specialised antigen-presenting cells (APCs), which links innate and adaptive immunity. The DCs constitute a complex system of cells found in peripheral tissues as well as immunological organs, and their presence was also documented in a vast range of human malignant tumours [20-25]. Response to cancer cells involves the capture of foreign antigens by immature DC subsets, their entailing maturation, migration to the lymph nodes and presentation of antigen peptides to $T$ cells via major histocompatibility

Address for correspondence: Grzegorz Dyduch MD, Chair of Pathomorphology, Jagiellonian Univeristy Medical College, 16 Grzegórzecka St, 31-351 Krakow, Poland, phone: +48 604406 920, e-mail: grzegorzdyduch@gmail.com Received: 8.01.2016, accepted: 22.04.2016. 
complex (MHC) molecules. However, depending on the biological context, DCs can either boost an effective host reaction to cancer cells or maintain local tissue tolerance that facilitates tumour growth [26, 27]. Distinct DC subtypes, each having a different phenotype, location and function can be identified on the basis of cell surface markers and intracellular molecules. The normal human skin contains epidermal Langerhans cells (LCS) and dermal DCs, while under pathological conditions, plasmacytoid as well as inflammatory dendritic epidermal cells happen to be detected [28].

Despite many studies on the issue of dendritic cells in various cancers, the underlying tumour-host interactions involving certain subsets has not yet been clearly elucidated, which prevents the translation of these immune factors into clinical use. Additionally, in the light of the importance of DCs, there is a very limited number of studies investigating the correlation between their density and histological characteristics of CMM [29-31].

\section{Aim}

The objective of the present study was to evaluate the infiltration of DCs expressing CD207/langerin, which is a C-type lectin displaying mannose-binding specificity, involved in ligand internalisation [32]. The study was conducted on a subset of 42 cases, which were obtained from patients diagnosed with melanoma in situ or invasive malignant melanoma of the skin. The number of DCs was analysed in relation to a degree of CMM progression and spatial location of DC infiltration (intratumoral, peritumoral, epidermal). Evaluation of these parameters might be helpful in explaining the prognostic and predictive value of DCs in the progression of CMM as well as their potential implementation in immunotherapeutical protocols.

\section{Material and methods}

\section{Material}

We used cutaneous tissue samples excised from $42 \mathrm{pa}$ tients who were diagnosed with melanoma in situ or invasive cutaneous melanoma of the skin between 2009 and 2013. The paraffin-embedded samples were obtained from the archives of the Department of Pathomorphology, while data concerning the subtype of lesion, melanoma stage (described using Breslow depth), presence of ulceration, mitotic activity (counted per $\mathrm{mm}^{2}$ ) and tumour regression features were collected from the database of the Department.

\section{Immunohistochemistry}

Immunohistochemistry was performed using the anti-human CD207 (Langerin) monoclonal antibody (clone 12D6, Novocastra, Leica Biosystems, Germany), dilution $1: 100$, incubation time $60 \mathrm{~min}$. Cutaneous tis- sue samples were stained manually and processed according to the protocol used on a routine basis in the laboratory of the Department of Pathomorphology. The selected paraffin-embedded tissue blocks were cut into $4 \mu \mathrm{m}$ thick sections, mounted on SuperFrost glass slides (ThermoScientific, USA) and dried in an incubator for $12 \mathrm{~h}$ in $34^{\circ} \mathrm{C}$. The obtained slides were deparaffinized, dehydrated and then incubated in $3 \% \mathrm{H}_{2} \mathrm{O}_{2}$ solution for 10 min to block endogenous peroxidase activity. Antigen retrieval was performed by immersing the slides in citrate buffer ( $\mathrm{pH} 6.0 ; 0.01 \mathrm{M}$ ) and subjecting them to $97^{\circ} \mathrm{C}$ in a water bath for $40 \mathrm{~min}$. Polyclonal secondary antibodies conjugated to horseradish peroxidase (Ultra Vision Quanto Detection System, Lab Vision, ThermoScientific, USA), using DAB (3,3'-diaminobenzidine) as chromogen, were applied to visualize the obtained antigen-antibody complexes. Cell nuclei were stained with hematoxylin to enhance contrast in tissue sections.

\section{Evaluation of immunostaining}

For each case quantitative assessment of CD207/ langerin-positive cells was performed under light microscopy. The positively stained cells (brown membranous staining) were counted at 400× magnification. For evaluation of intratumoral, peritumoral and intraepidermal cells, the CD207/langerin-positive cells were counted in tumour mass, along the tumour border and in overlying epidermis, respectively, within the whole section; then the cell count was recalculated per $\mathrm{mm}^{2}$ of tissue (depending on the number of investigated fields). The obtained results were entered into an Excel spreadsheet (Microsoft Corporation, USA).

\section{Statistical analysis}

Statistical data analysis was performed using Statistica 10 software (StatSoft, USA). The difference between the number of CD207/langerin-positive intratumoral, peritumoral or intraepidermal dendritic cells and type of lesion (invasive vs. in situ) were evaluated by using the Mann-Whitney U-test. Spearman rank and gamma correlation tests were performed to assess correlations between the numbers of CD207/langerin-positive cells (intratumoral, peritumoral and intraepidermal) and tumour stage (Breslow depth), presence of ulceration, mitotic activity and regression features. To evaluate the relationships between the content of CD207/langerinpositive cells and type of lesion, presence of ulceration or features of tumour regression, the $t$-test was performed. $P$-values $<0.05$ were considered significant.

\section{Results}

Among 42 cases investigated in our study, 25 (59.5\%) were diagnosed as invasive melanoma and 17 (40.5\%) as melanoma in situ. 


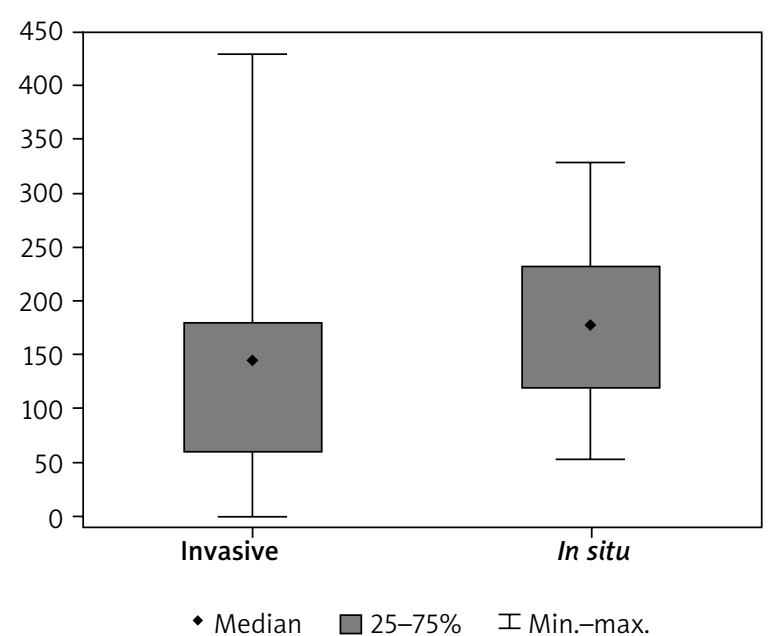

Figure 1. Density of langerin-positive cells in the epidermal compartment

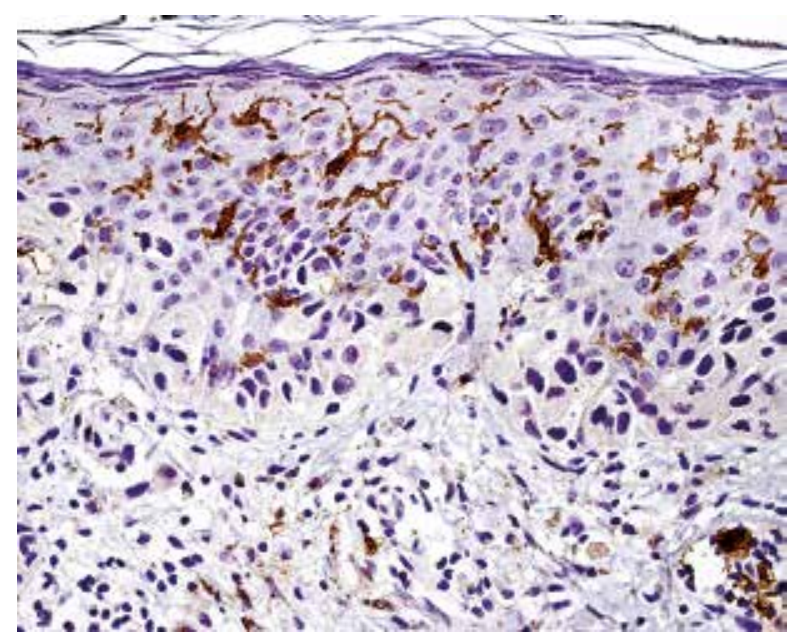

Figure 3. Numerous langerin-positive cells within epidermis in case of in situ melanoma

For all cases, average peritumoral CD207/langerinpositive cell count was $22.88($ SD $=28.15)$ and average epidermal CD207/langerin-positive cell count was 164.47 (SD = 101.15) (Figures 1 and 2).

There were statistically significant correlations between the number of peritumoral CD207/langerin-positive cells and Breslow depth $(R=0.60 ; p=0.002)$, mitotic activity $(R=0.62 ; p=0.001)$ as well as the presence of ulceration ( $R=0.58$; $p=0.002$ ) in invasive melanoma cases. There was a significantly $(p=0.002)$ higher number of peritumoral CD207/langerin-positive cells in ulcerated invasive melanoma samples (mean: 44.17, SD = $35.22)$ in comparison with invasive melanomas without ulceration (mean: 9.69, SD =9.40).

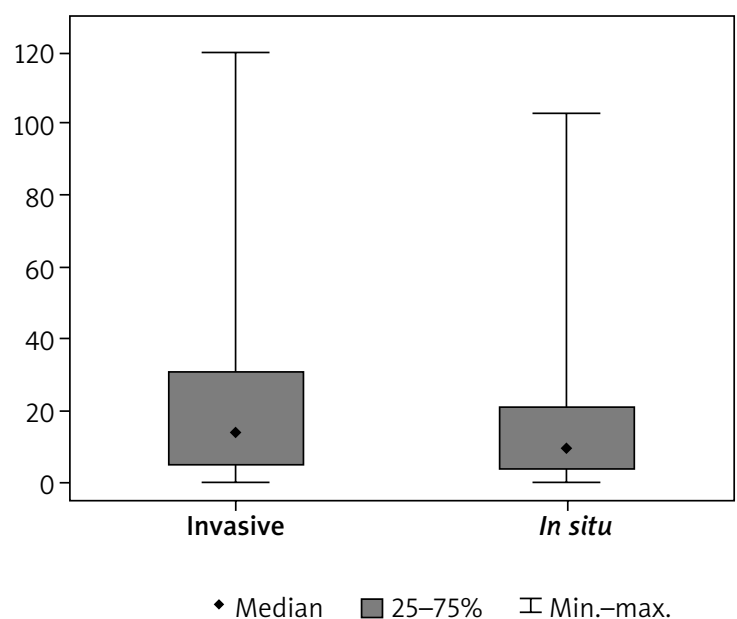

Figure 2. Density of langerin-positive cells in the peritumoral area

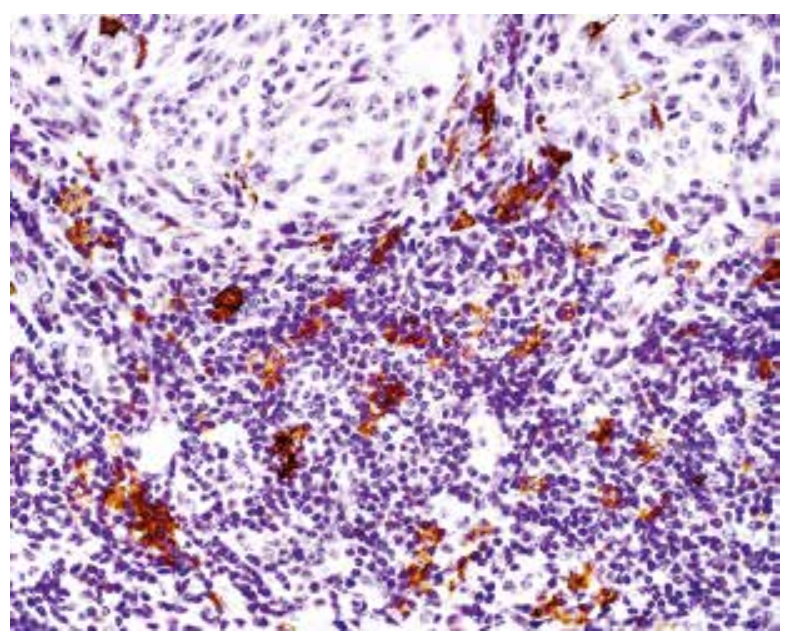

Figure 4. Langerin-positive cells at the tumour border in case of invasive melanoma

There was no statistically significant $(p>0.05)$ difference in peritumoral or intraepidermal CD207/langerin-positive cell count between invasive and in situ melanoma groups. Nonetheless, it is noteworthy that we observed a higher number of CD207/langerin-positive cells in the peritumoral area of invasive (mean: 26.24, SD $=30.36$ ) than of in situ lesions (mean: 17.94, SD = 24.59). Contrary to the above results, the number of intraepidermal CD207/langerin-positive cells in invasive melanomas was lower (mean: 150.78, SD = 114.18) than in in situ lesions (mean: 183.00, SD = 79.85) (Figures 3 and 4).

Among invasive melanomas, the average number of intratumoral CD207/langerin-positive cells (mean: 275.00, SD $=483.84$ ) and intraepidermal cells (mean: 173.20, SD $=156.57$ ) was higher in tumours with regres- 


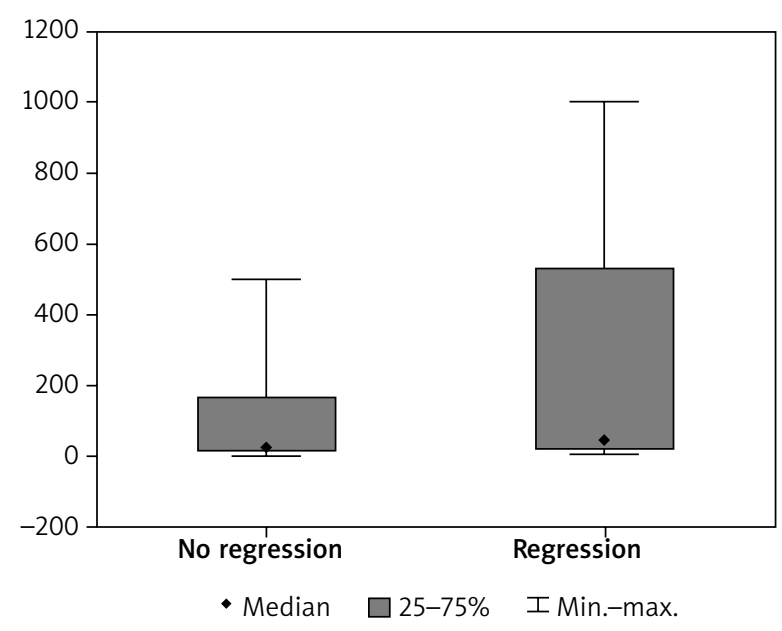

Figure 5. Density of intratumoral langerin-positive cells in invasive melanomas with and without regression

sion than in tumours without regression (mean: 95.32, $\mathrm{SD}=134.34$ - for intratumoral, mean: $148.35, \mathrm{SD}=106.41$ - for intraepidermal) (Figures 5 and 6).

By contrast, the peritumoral CD207/langerin-positive cell count was lower in invasive melanomas with regression (mean: $17.60, \mathrm{SD}=35.05)$ than in cases without regression (mean: 27.26, SD = 30.05). However, for all cases the differences were not statistically significant.

\section{Discussion}

Langerin (CD207) is a C-type lectin, binding glycosylated ligands in a calcium-dependent manner, and has many roles, such as in cell adhesion, endocytosis, natural killer-cell target recognition and dendritic-cell activation [33]. So far, it was generally assumed that Langerhans cells are the only cells expressing langerin [32, 34, 35], but now there is a growing body of evidence indicating that langerin expression is not confined to LCs exclusively as it is also found in dermal DCs [36-39] and other organs, such as lungs, liver, spleen and lymph nodes [36, 40]. In mouse skin, the population of DCs comprises CD207/ langerin-positive LCs, forming a network in epidermis, as well as CD207/langerin-positive and CD207/langerinnegative DC subsets residing in the dermis [37-39]. As far as human dermis is concerned, one study confirming the existence of CD207/langerin-positive dermal DCs has been published recently [36]. One of the main reasons why previous studies have evaluated dermis as langerin-negative compartment $[32,41]$ might be the fact that CD207/langerin-positive DCs found in the steady state in dermis are rare, and if they were ever noticed, they were regarded as LCs in transit through the dermis on their way to the lymph nodes [39, 42]. In addition, some in vitro studies have demonstrated that the production of langerin in human progenitor cells and monocytes can

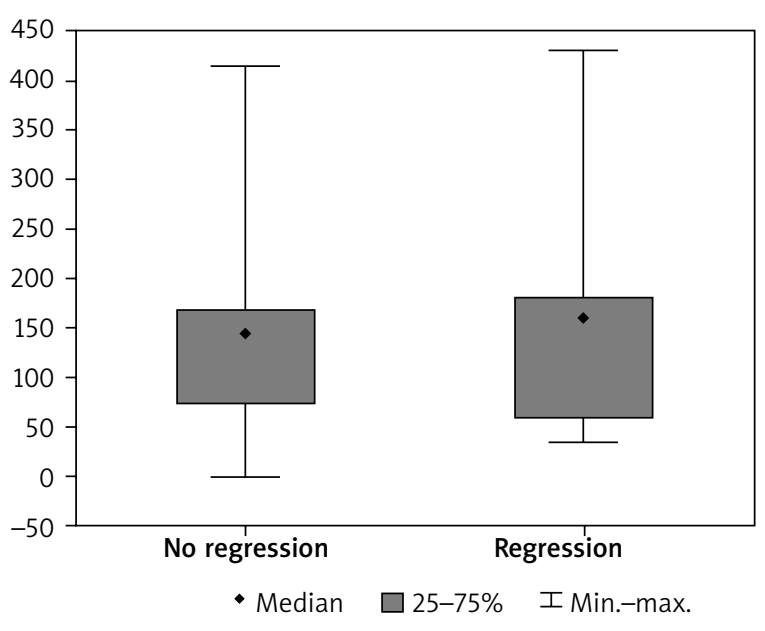

Figure 6. Density of epidermal langerin-positive cells in invasive melanomas with and without regression

be induced by a variety of factors, such as transforming growth factor $\beta$ (TGF- $\beta$ ), granulocyte-macrophage colony-stimulating factor (GM-CSF), tumor necrosis factor $\alpha$ (TNF- $\alpha$ ) and bone morphogenetic protein 7 (BMP7) [4345], which also supports the view that langerin might be expressed by distinct dermal DC subtype, not related to epidermal LCs. Although these observations need to be clarified, they have to be considered in the interpretation of the presented results.

Tumour thickness, mitotic rate and ulceration are the most dominant prognostic factors for clinical outcome of malignant melanoma [46]. Our study revealed a statistically significant positive correlation between each recognised prognostic feature and the infiltration of CD207/ langerin-positive DCs in the peritumoral area of invasive CMM. Since the increased tumour thickness, higher mitotic rate and presence of ulceration are indicators of decline in survival rates, our findings may suggest that a higher number of CD207/langerin-positive DCs at the border of the tumour favours disease progression and is associated with worse prognosis. This conclusion would be consistent with the observation that CD207/langerinexpressing dendritic cells in human tissues are related to immature CD1a/CD1c-positive cells [36], and thus presumably play a role in tolerance rather than in immunity, as immature DCs are unable to effectively present processed foreign antigens to T cells. It is, however, still unknown whether the higher infiltration of immature DCs in peritumoral area is mostly the consequence of their impaired maturation or is solely caused by the excessive migration to the pathologically altered site, and the progression of the disease should be rather ascribed to the inadequate functioning of mature dendritic cells. If a case of massive migration was considered to be more probable, it would be recommended to investigate local factors, which may possibly support the recruitment of 
CD207/langerin-positive dendritic cells in CMM, as e.g. CCL20 chemokine was proposed to be crucial for CD207/ langerin-positive DCs migration to EBV-associated tumours [47]. There are also substantial ambiguities resulting from the lack of sufficient information about the pathophysiological processes involved in CMM progression, which makes the speculation about the role of DCs in the disease progression even more complicated; for instance, very little is known about the underlying biological mechanisms of CMM ulceration [48]. Literature data concerning the significance of CD207/langerinpositive cells in CMM are very scarce and findings vary considerably between papers. In a study conducted on 185 primary melanomas, Gerber et al. did not show any significant influence of CD207/langerin expression on the clinical outcome [49]. Moreover, given the observation that in human tissues CD207/langerin-positive DCs were found to be closely related to CD1a-positive subsets, as described above, it is justified to mention that infiltration of CD1a-positive DC peritumoral area of CMM was observed to correlate positively with decreased tumour thickness [50], which might be interpreted as being contrary to our findings. In addition, we have found a significant inverse association between the number of CD207/langerin-positive DCs and the presence of melanoma regression features, whose presence is traditionally regarded as an indicator of poor prognosis. Nonetheless, the impact of tumour regression is still unclear, as some studies revealed that this feature does not have any independent prognostic value [51], while others demonstrated a higher metastatic rate in CMMs with extensive regression [52]. Taken together, there are still many unresolved questions, which prevents us from drawing valid conclusions on whether CD207/langerin-positive cells play any specific role in inducing or regulating an immune response in the progression of CMM, and thus their correlations with established prognostic factors must be interpreted with caution.

We found a substantially higher average number of intratumoral and epidermal CD207/langerin-positive DCs in invasive melanomas with regression. With regard to the differences in the spatial distribution of CD207/ langerin-positive DCs we can conclude that despite the enhanced intranestal recruitment of DCs in more advanced stages, the immune interactions are ineffective to elicit an appropriate antitumor response. It is suggested that some melanoma-derived factors, such as TGF- $\beta$ and interleukin 10 (IL-10), found in melanoma cell nests, might adversely influence migration of dendritic cells, their differentiation and function [53-55]. Neagu et al. demonstrated that the presence of LCs within melanoma tumour mass correlates with the Breslow index but not with ulceration; yet the general intratumoral LC content was described to be rather scarce [8]. Regarding other human malignancies, the infiltration of CD207/ langerin-positive DCs was also observed in Hodgkin lymphoma, nasopharyngeal carcinoma, oral squamous cell carcinoma and breast cancer [47, 56, 57]. However, in the latter case the infiltration by immature CD207/ langerin-positive DCs was found in only one-third of the breast cancer tissues and did not correlate with clinicopathological data. In our cohort of melanoma samples, we have also observed a higher intratumoral langerin expression compared to the peritumoral area in invasive CMM. Assuming again that langerin is mostly present on immature dendritic cells and is related to CD1a-positive subsets, this observation is in agreement with the results obtained by other authors investigating various human malignancies, as it is generally suggested that immature dendritic cells are located within tumour nests, while mature subsets reside in peritumoral compartment [29, 58]. Conversely, the intraepidermal infiltration of CD207/ langerin-positive DCs was more pronounced in non-invasive lesions of our study, which is in concert with the findings of other authors showing a reduced number of intraepidermal DCs in invasive melanoma tissues [53, 59]. The possible explanations of the depletion of DCs in the epidermis during the evolution of CMM include necrosis, regression and scarring in more advanced stages of the tumour [53] as well as the presence of basement membrane along with the lack of epidermal own blood vessels. We represent the view that in order to better understand the role of CD207/langerin-positive DCs in CMM evolution, the issue of their uneven distribution between invasive and non-invasive melanomas should be revisited on a larger sample of cases.

\section{Conclusions}

Our results may shed light on the relevance of CD207/langerin-positive DCs at different stages of CMM and clarify some of their associations with traditional risk prediction based on histopathological characteristics. In interpreting these findings we should, however, remember that the evaluation of density and location of DCs relied on staining for CD207/langerin exclusively, thus, we were unable to analyse the whole spectrum of DC subsets and their interactions with other immune cells involved in melanoma carcinogenesis. In order to draw a complete picture of the role of DCs in melanoma control, the thorough analysis of the dendritic cell family, including numerous markers, as well as other presumably contributing microenvironmental factors, such as cytokines, is required. Moreover, the inclusion of melanoma metastases might also bring some added value to the evaluation of DCs in progression of the disease. In addition, we should also bear in mind that melanoma patient's survival can vary significantly among individuals within the same tumour stage and widely accepted classifications (e.g. Breslow depth, TNM) may not utterly correspond with the ongoing immunological processes. Thus, only a prospective analysis of the patient's clinical 
outcome will allow us to better understand the predictive and prognostic significance of CD207/langerin-positive tumour-infiltrating DCs. Although the role of DCs infiltrating CMM demands further elucidation, we believe that our study gives a valuable insight into the role of CD207/langerin-positive DCs in the progression of CMM and might prove useful in incorporating immune scoring in clinical settings.

\section{Conflict of interest}

The authors declare no conflict of interest.

\section{References}

1. Parkin DM, Whelan SL, Ferlay J, et al. Cancer Incidence in Five Continents. IARC Scientific Publications, 143, Lyon 1997.

2. Curado MP, Edwards B, Shin HR, et al. Cancer Incidence in Five Continents. IARC Scientific Publications, 160, Lyon 2007.

3. Merrill SJ, Ashrafi S, Subramanian M, et al. Exponentially increasing incidences of cutaneous malignant melanoma in Europe correlate with low personal annual UV doses and suggests 2 major risk factors. Dermatoendocrinol 2015; 7: e1004018.

4. Steliarova-Foucher E, O'Callaghan M, Ferlay J, et al. European cancer observatory: cancer incidence, mortality, prevalence and survival in Europe. European Network of Cancer Registries, International Agency for Research on Cancer 2012; Version 1.0.

5. Bosetti C, Bertuccio P, Malvezzi M, et al. Cancer mortality in Europe, 2005-2009, and an overview of trends since 1980. Ann Oncol 2013; 24: 2657-71.

6. Rauschenberg R, Garzarolli M, Dietrich U. Systemic therapy of metastatic melanoma. J Dtsch Dermatol Ges 2015; 13: 1223-37.

7. González-Cao M, Rodón J, Karachaliou N, et al. Other targeted drugs in melanoma. Ann Transl Med 2015; 3: 266.

8. Neagu M, Constantin C, Zurac S. Immune parameters in the prognosis and therapy monitoring of cutaneous melanoma patients: experience, role, and limitations. Biomed Res Int 2013; 2013: 107940.

9. Mhawech-Fauceglia P, Wang D, Ali L, et al. Intraepithelial $T$ cells and tumor-associated macrophages in ovarian cancer patients. Cancer Immun 2013; 13: 1.

10. Domingues P, González-Tablas M, Otero Á, et al. Tumor infiltrating immune cells in gliomas and meningiomas. Brain Behav Immun 2016; 53: 1-15.

11. Ostuni R, Kratochvill F, Murray PJ, et al. Macrophages and cancer: from mechanisms to therapeutic implications. Trends Immunol 2015; 36: 229-39.

12. Ladányi A. Prognostic and predictive significance of immune cells infiltrating cutaneous melanoma. Pigment Cell Melanoma Res 2015; 28: 490-500.

13. Lepore M, de Lalla C, Mori L, et al. Targeting leukemia by CD1c-restricted T cells specific for a novel lipid antigen. Oncoimmunology 2014; 4: e970463.

14. Prue RL, Vari F, Radford KJ, et al. A phase I clinical trial of CD1c (BDCA-1)+ dendritic cells pulsed with HLA-A*0201 peptides for immunotherapy of metastatic hormone refractory prostate cancer. J Immunother 2015; 38: 71-6.

15. Mac Keon S, Ruiz MS, Gazzaniga S, et al. Dendritic cell-based vaccination in cancer: therapeutic implications emerging from murine models. Front Immunol 2015; 6: 243.
16. Qiu L, Li J, Yu S, et al. A novel cancer immunotherapy based on the combination of a synthetic carbohydrate-pulsed dendritic cell vaccine and glycoengineered cancer cells. Oncotarget 2015; 6: 5195-203.

17. Castillo-Montiel E, Chimal-Eguía JC, Tello Jl, et al. Enhancing dendritic cell immunotherapy for melanoma using a simple mathematical model. Theor Biol Med Model 2015; 12: 11.

18. Van Lint S, Wilgenhof S, Heirman C, et al. Optimized dendritic cell-based immunotherapy for melanoma: the TriMixformula. Cancer Immunol Immunother 2014; 63: 959-67.

19. Benencia F, Sprague L, McGinty J, et al. Dendritic cells the tumor microenvironment and the challenges for an effective antitumor vaccination. J Biomed Biotechnol 2012; 2012: 425476.

20. Bell D, Chomarat P, Broyles D, et al. In breast carcinoma tissue, immature dendritic cells reside within the tumor, whereas mature dendritic cells are located in peritumoral areas. J Exp Med 1999; 190: 1417-26.

21. Schwaab T, Weiss JE, Schned AR, et al. Dendritic cell infiltration in colon cancer. J Immunother 2001; 24: 130-7.

22. Lu L, Pan K, Zheng HX, et al. IL-17A promotes immune cell recruitment in human esophageal cancers and the infiltrating dendritic cells represent a positive prognostic marker for patient survival. J Immunother 2013; 36: 451-8.

23. Remark R, Becker C, Gomez JE, et al. The non-small cell lung cancer immune contexture. A major determinant of tumor characteristics and patient outcome. Am J Respir Crit Care Med 2015; 191: 377-90.

24. Reichert TE, Scheuer C, Day R, et al. The number of intratumoral dendritic cells and zeta-chain expression in T cells as prognostic and survival biomarkers in patients with oral carcinoma. Cancer 2001; 91: 2136-47.

25. Upadhyay J, Rao NN, Upadhyay RB. A comparative analysis of Langerhans cell in oral epithelial dysplasia and oral squamous cell carcinoma using antibody CD-1a. J Cancer Res Ther 2012; 8: 591-7.

26. Karthaus N, Torensma R, Tel J. Deciphering the message broadcast by tumor-infiltrating dendritic cells. Am J Pathol 2012; 181: 733-42.

27. Zitvogel L, Tesniere A, Kroemer G. Cancer despite immunosurveillance: immunoselection and immunosubversion. Nat Rev Immunol 2006; 6: 715-27.

28. Udey MC. Skin dendritic cells in immunity and autoimmunity. I Investig Dermatol Symp Proc 2004; 9: 15-7.

29. Vermi W, Bonecchi R, Facchetti F, et al. Recruitment of immature plasmacytoid dendritic cells (plasmacytoid monocytes) and myeloid dendritic cells in primary cutaneous melanomas. J Pathol 2003; 200: 255-68.

30. Ladányi A, Kiss J, Somlai B, et al. Density of DC-LAMP(+) mature dendritic cells in combination with activated T lymphocytes infiltrating primary cutaneous melanoma is a strong independent prognostic factor. Cancer Immunol Immunother 2007; 56: 1459-69.

31. Jensen TO, Schmidt H, Møller HJ, et al. Intratumoral neutrophils and plasmacytoid dendritic cells indicate poor prognosis and are associated with pSTAT3 expression in AJCC stage I/II melanoma. Cancer 2012; 118: 2476-85.

32. Valladeau J, Ravel O, Dezutter-Dambuyant C, et al. Langerin, a novel C-type lectin specific to Langerhans cells, is an endocytic receptor that induces the formation of Birbeck granules. Immunity 2000; 12: 71-81.

33. Merad M, Ginhoux F, Collin M. Origin, homeostasis and function of Langerhans cells and other langerin-expressing dendritic cells. Nat Rev Immunol 2008; 8: 935-47. 
34. Hunger RE, Sieling PA, Ochoa MT, et al. Langerhans cells utilize CD1a and langerin to efficiently present nonpeptide antigens to T cells. J Clin Invest 2004; 113: 701-8.

35. Valladeau J, Duvert-Frances V, Pin JJ, et al. The monoclonal antibody DCGM4 recognizes langerin, a protein specific of Langerhans cells, and is rapidly internalized from the cell surface. Eur J Immunol 1999; 29: 2695-704.

36. Bigley $V$, McGovern N, Milne $P$, et al. Langerin-expressing dendritic cells in human tissues are related to CD1C+ dendritic cells and distinct from Langerhans cells and CD141high XCR1+ dendritic cells. J Leukoc Biol 2015; 97: 627-34.

37. Bursch LS, Wang L, Igyarto B, et al. Identification of a novel population of Langerin+ dendritic cells. J Exp Med 2007; 204: 3147-56.

38. Poulin LF, Henri S, de Bovis B, et al. The dermis contains langerin+ dendritic cells that develop and function independently of epidermal Langerhans cells. J Exp Med 2007; 204: 3119-31.

39. Ginhoux F, Collin MP, Bogunovic M, et al. Blood-derived dermal langerin+ dendritic cells survey the skin in the steady state. J Exp Med 2007; 204: 3133-46.

40. Takahara K, Omatsu Y, Yashima Y, et al. Identification and expression of mouse Langerin (CD207) in dendritic cells. Int Immunol 2002; 14: 433-44.

41. Angel CE, Lala A, Chen CJ, et al. CD14+ antigen-presenting cells in human dermis are less mature than their CD1a+ counterparts. Int Immunol 2007; 19: 1271-9.

42. Romani N, Clausen BE, Stoitzner P. Langerhans cells and more: langerin-expressing dendritic cell subsets in the skin. Immunol Rev 2010; 234: 120-41.

43. Caux C, Dezutter-Dambuyant C, Schmitt D, et al. NF-alpha cooperate in the generation of dendritic Langerhans cells. Nature 1992; 360: 258-61.

44. Strobl H, Riedl E, Scheinecker C, et al. TGF-beta 1 promotes in vitro development of dendritic cells from CD34+ hemopoietic progenitors. J Immunol 1999; 157: 1499-507.

45. Yasmin N, Bauer T, Modak M, et al. Identification of bone morphogenetic protein 7 (BMP7) as an instructive factor for human epidermal Langerhans cell differentiation. J Exp Med 2013; 210: 2597-610.

46. Balch CM, Gershenwald JE, Soong SJ, et al. Final version of 2009 AJCC melanoma staging and classification. J Clin Oncol 2009; 27: 6199-206.

47. Braz-Silva PH, Vitale S, Butori C, et al. Specific infiltration of langerin-positive dendritic cells in EBV-infected tonsil, Hodgkin lymphoma and nasopharyngeal carcinoma. Int J Cancer 2011; 128: 2501-8.

48. Bønnelykke-Behrndtz LM, Schmidt H, Damsgaard TE, et al. Consumption of the epidermis: a suggested precursor of ulceration associated with increased proliferation of melanoma cells. Am J Dermatopathol 2015; 37: 841-5.

49. Gerber AL, Münst A, Schlapbach C, et al. High expression of FOXP3 in primary melanoma is associated with tumour progression. Br J Dermatol 2014; 170: 103-9.

50. Ladányi A, Kiss J, Somlai B, et al. Density of DC-LAMP(+) mature dendritic cells in combination with activated T lymphocytes infiltrating primary cutaneous melanoma is a strong independent prognostic factor. Cancer Immunol Immunother 2007; 56: 1459-69.

51. Eriksson H, Frohm-Nilsson M, Järås J, et al. Prognostic factors in localized invasive primary cutaneous malignant melanoma: results of a large population-based study. Br J Dermatol 2015; 172: 175-86.
52. Ronan SG, Eng AM, Briele HA, et al. Thin malignant melanomas with regression and metastases. Arch Dermatol 1987; 123: 1326-30.

53. Stene MA, Babajanians M, Bhuta S, et al. Quantitative alterations in cutaneous Langerhans cells during the evolution of malignant melanoma of the skin. J Invest Dermatol 1988; 91: 125-8.

54. Conrad CT, Ernst NR, Dummer W, et al. Differential expression of transforming growth factor beta 1 and interleukin 10 in progressing and regressing areas of primary melanoma. J Exp Clin Cancer Res 1999; 18: 225-32.

55. Enk AH, Jonuleit H, Saloga J, et al. Dendritic cells as mediators of tumor-induced tolerance in metastatic melanoma. Int J Cancer 1997; 73: 309-16.

56. O’Donnell RK, Mick R, Feldman M, et al. Distribution of dendritic cell subtypes in primary oral squamous cell carcinoma is inconsistent with a functional response. Cancer Lett 2007; 255: $145-52$.

57. Treilleux I, Blay JY, Bendriss-Vermare N, et al. Dendritic cell infiltration and prognosis of early stage breast cancer. Clin Cancer Res 2004; 10: 7466-74.

58. Bell D, Chomarat P, Broyles D, et al. In breast carcinoma tissue, immature dendritic cells reside within the tumor, whereas mature dendritic cells are located in peritumoral areas. J Exp Med 1999; 190: 1417-26.

59. Toriyama K, Wen DR, Paul E, et al. Variations in the distribution, frequency, and phenotype of Langerhans cells during the evolution of malignant melanoma of the skin. J Invest Dermatol 1993; 100: 269S-73S. 